\title{
Evidencia actual de la influencia del cambio climático en la variable lluvia diaria observada en Honduras a partir de la base de datos disponible a la fecha
}

\author{
Roberto Ávalos, Hugo Medina, Yolanda Fletes, Gladys Rojas ${ }^{1}$
}

\section{RESUMEN}

El motivo de la investigación es descubrir evidencia de que la lluvia diaria observada en el territorio hondureño está siendo afectada por el cambio climático; para este propósito se utilizará como data base las series diarias de la información disponible a la fecha.

El territorio hondureño desde hace algunos años ha estado siendo afectado por fenómenos meteorológicos extremos máximos que han perjudicado en las diferentes áreas de la sociedad hondureña.

Modelos atmosféricos globales para estudiar la influencia del cambio climático han sido aplicados en Honduras, cuyos resultados son proyecciones, en el tiempo, del comportamiento climático basado en ciertos supuestos. Tales resultados son interesantes para tomar en cuenta por los encargados de la planificación del país.

En la presente investigación se han utilizado datos de lluvia diaria recolectada en toda la red de estaciones medidoras de lluvia que existen en el territorio hondureño, desde el 1944 al 2013; cabe mencionar que el inicio de las mediciones es diferente en cada estación. Esta red está conformada por subredes perteneciente a la Empresa Nacional de Energía Eléctrica, Dirección de Recursos Hídricos, Servicio Meteorológico Nacional, Servicio Autónomo Nacional de Agua y Alcantarillado y Universidad Nacional Autónoma de Honduras. Las series de lluvia diaria recolectadas tienen diferentes longitudes, por lo cual han sido clasificadas por cantidad de años disponible, a fin de utilizar la mayor cantidad de datos. Análisis puntuales y espaciales fueron aplicados para conocer la calidad de los mismos.

\footnotetext{
${ }^{1}$ Beneficiarios de una beca sustantiva de la DICYP, Grupo de Investigación del Departamento de Ingeniería Civil, Facultad de Ingeniería, UNAH: robertofredy@yahoo.com.
} 
Mediante simple regresión se investigó la tendencia de la lluvia diaria de los promedios de 3, 5, 10, 20, 30, 90, 180, 365, 1025, 1825 y 3650 días; estos promedios fueron determinados mediante la media aritmética y la media móvil. También se investigaron las tendencias de los días sin lluvia para las series de 365 días.

En el estado actual de la información disponible de lluvia diaria, todavía no hay evidencia bien definida sobre la tendencia de aumento o disminución de la misma.

Palabras claves: longitud de serie, media aritmética, media móvil, tendencia y días sin Iluvia.

\section{ABSTRACT}

The Honduran territory since some years ago has been affected by extreme weather events maximum that have harmed the Honduran economy; leaving strong negative impact on the society and painful memories of deceased persons. Global atmospheric models to study the influence of climate change have been applied in Honduras; the results obtained are projections at the time of the climatic behavior based on certain assumptions. Interesting results to be taken into account by those responsible for the planning of the country.

In the present research we have used daily rainfall data collected in the entire network of stations feeler rain that exists in the Honduran territory, since the 2013 toward years ago; that is to say the entire series available. The series of daily rainfall obtained have different lengths; by which we have classified these series in different lengths, in order to use the increased amount available data. Punctual and spatial analysis were applied to know the quality of the same.

Using simple regression was investigated the trend of daily rainfall averages of 3,5 , $10,20,30,90,180,365,1025,1825$ and 3650 days; these averages were determined by the arithmetic mean and the moving average. It was also investigated the trends of the days without rain for the series of 365 days.

The influence of climate change in the rain to daily resolution and as quantity not still displays well-defined evidence on the linear trend of increase or decrease in the size of the variable. The slopes of the trends are very low to define significantly the increase or decrease of the rain. 


\section{INTRODUCCIÓN}

En estos últimos 20 años han ocurrido en Honduras una serie de fallas estructurales en varias construcciones de ingeniería, por ejemplo puentes, carreteras, urbanizaciones y cajas; dichas fallas causan impactos negativos a la economía hondureña. Actualmente, esta situación fácilmente puede ser justificada por la influencia del cambio climático.

Sobre el cambio climático muchos investigadores han identificado, mediante observaciones de varios años, incrementos positivos de la variable temperatura (Environnement Canadá, 2010). Básicamente este aumento es el resultado de la actividad humana que está provocando efectos dañinos en muchos lugares de la tierra y los investigadores sobre el tema recomiendan disminuir la emisión de gases (OMM Conferencia Mundial sobre el Clima, 1986 y Convención Mundial sobre Cambio Climático, 1992).

Muchas son las variables climáticas que participan en la caracterización del clima, tales como temperatura, lluvia, evaporación, horas de sol, etc. En nuestro caso en particular, es la cantidad de lluvia diaria observada y medida en el terreno en unidades de $\mathrm{mm}$, la que es objeto de nuestra investigación, afín de determinar la influencia del cambio climático en dicha variable. Es importante mencionar que la presente investigación forma parte de un marco de investigaciones sobre las principales variables climáticas que utiliza el ingeniero civil en las diferentes etapas que conlleva la construcción de las obras civiles.

Además, es necesario construir fundamentos científicos que sustenten la idea de ajustar fórmulas y procedimientos que comúnmente utiliza el ingeniero en su labor, con el objetivo de adaptarse al cambio climático. Esto es lo que ha motivado la realización de la presente investigación, cuya finalidad principal es determinar, a partir de las series de lluvia diaria existente en el territorio hondureño, el efecto del cambio climático en la cantidad de lluvia; es decir, si estos cambios climáticos han provocado, a la fecha actual, aumento o disminución de la lluvia únicamente en su dimensión de cantidad.

La resolución de la lluvia seleccionada en la presente investigación es la diaria (mm/día); esto es congruente con lo expresado por Daily dataset of 20Th-Century surface air temperature and precipitation series for the European Climate Assessment. Wiley Intersciences (Royal-Meteorological-Society. 2002), en donde se dice que hay un 
creciente interés en los fenómenos meteorológicos extremos y que la identificación de cambios requiere de series de tiempo preciso, completa y espacialmente coherente y al menos de una resolución diaria.

Es importante indicar que el territorio hondureño es influenciado por diferentes fenómenos meteorológicos continentales que conjuntamente con el relieve caracterizan su clima: es ruta de huracanes, ondas tropicales, sistemas de baja presión, ciclones tropicales, vaguadas, tormentas tropicales, frentes fríos, El Niño, La Niña, etc. Asimismo, las estaciones del año no están bien definidas. De tal forma que se habla del periodo con meses lluviosos y periodo con meses secos; igualmente, el inicio y fin de dichos periodos no es el mismo en todo el territorio.

Actualmente Honduras dispone de una red de estaciones en donde se mide la variable lluvia; esta red cubre lo largo y ancho del país. El manejo de la red está a cargo, principalmente, de las instituciones gubernamentales quienes se responsabilizan del mantenimiento, recolección y almacenamiento de los datos. Las series de datos de lluvia disponibles tienen longitudes diferentes, de las cuales se seleccionaron las más largas. Análisis gráficos de calidad de datos, puntual y espacial, se aplicaron a los datos seleccionados. La media aritmética y la media móvil para diferentes periodos de días permitieron obtener los datos para el análisis de tendencia. La metodología de tendencia lineal fue el procedimiento utilizado para encontrar, a la fecha actual, evidencia del cambio climático en la variable lluvia diaria.

\section{ANTECEDENTES}

El comportamiento del sistema atmosférico: tierra, océanos y capas de hielo, condicionan el clima del globo terrestre. Esto significa que cualquier cambio que afecte este sistema tiene repercusiones en el clima y en todas las consecuencias que implica.

La fundación y progreso de las sociedades humanas y la acción de las fuerzas naturales pueden cambiar notablemente, en regiones vastas, el estado de la superficie, la distribución del agua y los grandes movimientos del aire. Tales efectos son capaces de hacer variar, en el curso de muchos siglos, el grado promedio del calor, debido a que las expresiones analíticas contienen coeficientes que se relacionan con el estado de la superficie y el cual influye en gran medida a la temperatura (Fourier, 1827). 
Con base a investigaciones realizadas sobre la absorción de la radiación infrarroja en diferentes gases, se sabe que los gases en la atmósfera como vapor de agua, hidrocarburos como metano y dióxido de carbono, bloquean en gran manera la radiación, influenciado la temperatura (Tyndall, 1864). De esta información surgieron varios planteamientos por algunos investigadores.

La investigación sobre los gases continuaron, de tal forma que se llegó a cuantificar las fuentes naturales de $\mathrm{CO} 2$ y se encontró que el estimado de la producción de carbón de fuentes industriales era comparable con las fuentes naturales, que esta emisión de gases de carbono llevaría al calentamiento del planeta y que esto tomaría miles de años (Hogbom, 1890; Arthenus, 1896).

Los descubrimientos en las investigaciones avanzaron, al grado que se afirma que en ausencia de regeneraciones desconocidas, tales como cambio en las nubes y la duplicación de los niveles de dióxido de carbono actuales, esto daría lugar al aumento de aproximadamente $2^{\circ} \mathrm{C}$ en la temperatura global (Manabe \& Wetherald, 1967).

La contaminación humana representa una amenaza importante a la seguridad internacional y está provocando consecuencias dañinas sobre muchas partes del globo terráqueo. La OMM declaró que en el 2005 el mundo debe disminuir sus emisiones en un 20 \% por debajo de los niveles de 1988 (OMM, 1988). En la Convención Mundial sobre Cambio Climático realizada en Kioto, Japón, en 1992, se puso de manifiesto el papel importante que juegan las emisiones de gases de invernadero en el cambio climático y la necesidad de reducirlos estableciendo reglas para su disminución.

Hasta mediado de 1985, existían en Honduras reglas establecidas para definir la fecha de inicio de la época lluvia, la fecha de la presentación y finalización de la canícula y fecha de finalización del periodo lluvioso. También se tenían bien determinados los periodos de duración del fenómeno de El Niño. Pocos años después se habló sobre la aparición de La Niña. Actualmente las cosas ya no son así y hay mucha incertidumbre en el establecimiento de dichas reglas (Zúniga, 1989 y 1990). La influencia del fenómeno de El Niño en Honduras se manifiesta en una disminución de la lluvia en la mayor parte del país y bajo el efecto de La Niña es todo lo contrario, se observa comúnmente en eventos extremos máximos en diferentes partes del país (Zúniga, 1989 y 1990).

El efecto del cambio climático no solamente influencia a la temperatura, posiblemente también afecta a la variable precipitación. Existe una incertidumbre con respecto a 
los cambios en precipitación a nivel global, aunque hay evidencia de que fenómenos como El Niño, que perjudica la lluvia a escala continental, ya está presentando anomalías (Leverman \& O'Brien, 1991). En Argentina, durante el episodio del evento El Niño, hay una tendencia a disminuir la precipitación media en el año antes del evento y se extiende hasta marzo del año del evento. Manifestándose también que en una vasta región al este de los Andes, la más fuerte anomalía de precipitación positiva se produce en la primavera de 1999 y que durante el verano las anomalías de precipitación positiva casi desaparecen (Grimm, 1999).

La calidad de los datos climáticos, puntual y espacial, son básicos para poder llegar a identificar cambios en el comportamiento de las variables y al menos con una resolución diaria según Jones (1999); el autor menciona también que Europa dispone de una buena base de datos climáticos mensuales, pero que hasta el momento no están disponibles los datos diarios.

Los fenómenos extremos son situaciones de mucho interés en la ingeniería, ya que afectan la producción de alimentos, olas de frío, calor severo y vendavales (Easterling, 2000; Houghton, 2001). En Europa, partiendo de una base de datos diarios de variables climáticas recolectada por el Grupo Evaluación Climática de Europa (ECA), se muestra que durante los meses de invierno de 1976 a 1999 hay un aumento positivo del calentamiento en Europa con relación al número de días de calentamiento en la mayoría de las estaciones; pero no se encontró una tendencia negativa en el número de días de frío. Muestra también que las precipitaciones de invierno ocurridas entre 1946 y 1999 que las tendencias positivas prevalecen en las áreas que son más secas y húmedas (Royal Meteorological Society, 2002).

Investigaciones realizadas en Italia con datos mensuales de los dos últimos 200 años muestran que hay una tendencia decreciente de la variable lluvia (5\%), aunque baja y raramente significativa (Brunetti, Mauger, Monti \& Nannia, 2006). Igualmente se menciona que ha habido una disminución de la precipitación en las regiones subtropicales y tropicales y el aumento de la precipitación en las latitudes altas (Trenberth, 2007).

En el caso de Canadá y con relación a la precipitación nacional ocurrida en el 2010 en este país, se presentó un verano más húmedo que lo normal con precipitaciones de $4.2 \%$ por encima de la normal. El verano más lluvioso fue en el 2005 , con precipitaciones del $21 \%$ por encima de lo normal y el más seco fue en el verano de 1958, con precipitaciones del $14 \%$ por debajo de la normal. A nivel de todo Canadá las anomalías en los veranos de 1948 a 1972 tienden a ser negativas en su mayor parte 
y a partir de 1973 a 2010, tienden en su mayor parte a ser positivas (Environnement Canadá, 2010).

Es importante mencionar que existe en la comunidad científica internacional una serie de modelos de circulación general (GCM) para pronosticar a largo plazo y bajo condiciones supuestas de nXCO2, el comportamiento de la temperatura y la lluvia. Se tiene conocimiento de que en Centroamérica ha sido aplicado en Costa Rica y Honduras.

En Honduras, se han hecho escenarios climáticos hasta 2090, en donde expresan cómo sería el clima en esos tiempos bajo condiciones de un mundo heterogéneo con fuertes crecimiento poblacional y un lento desarrollo económico y tecnológico, también con una población que aumenta a un ritmo menor que la condición anterior y con desarrollo económico intermedio y un cambio tecnológico menos rápido y más diverso (Argeñal, 2010). En Honduras, durante la temporada lluviosa influenciada por la ocurrencia de El Niño moderado, hay una ligero aumento de la lluvia mensual en el mes de junio en la zona occidental del país, también en la zona central, en la zona oriental, zona sur, noreste de El Paraíso y este de Olancho. En cambio, en la zona este de la costa norte se presenta una disminución de la lluvia mensual (Argeñal, 2010).

Toda esta problemática del cambio climático sobre su influencia positiva o negativa en la magnitud de la lluvia todavía no está bien definida. Varias investigaciones a nivel de dato mensual se han hecho y muy poco a nivel de resolución diaria. Honduras tiene un clima tropical, dispone de una serie de datos de lluvia diaria con diferentes longitudes y están distribuidas en diferentes partes del país, de modo que investigar sobre la influencia del cambio climático en las magnitudes de la lluvia diaria se convierte para nuestra investigación en un reto.

\section{METODOLOGÍA}

La evidencia de aumento o disminución de la lluvia como cantidad, a resolución diaria, será determinada mediante regresión lineal mediante el tipo lluvia promedio = $\mathrm{f}$ (tiempo); en donde la pendiente caracterizada por su magnitud y su signo (+ 0 -) indicará el aumento o disminución de la lluvia.

La lluvia promedio en mm/día y el tiempo (días) variará entre 3, 5, 10, 20, 30, 90, 180, 
$365,1025,1825$ y 3650 días. Las lluvias promedios serán determinadas mediante los métodos conocidos como la media aritmética y la media móvil, con el fin de cubrir la variabilidad de la lluvia en diferentes grupos de tiempo.

La lluvia es una variable natural que cae sobre el territorio hondureño y el fuerte relieve que caracteriza a este país, conjuntamente con el viento y su dirección (el viento es una variable natural), modifica la distribución de la misma. Las estaciones medidoras instaladas en el terreno miden la cantidad de lluvia como resultado final de lo mencionado. Por consiguiente, la presente investigación se encuentra dentro de la categoría de investigación descriptiva longitudinal.

El espacio de investigación es el territorio hondureño cubierto por la red de estaciones medidoras de cantidad de lluvia diaria a través del tiempo. Las longitudes de las series de datos disponibles son variables, ya que las mediciones no se iniciaron en la misma fecha. Después de los análisis de calidad de datos, las estaciones con datos de más 30 años y las estaciones entre 20 y 30 años fueron las utilizadas para realizar la investigación.

Cabe también mencionar que la red de estaciones medidoras de lluvia está conformada por estaciones convencionales y estaciones telemétricas. Las series de datos disponibles de las telemétricas son de periodo muy corto y además presentan mucha discontinuidad, lo que dificulta su utilización. Para la investigación se consideraron únicamente los datos de las estaciones convencionales manejadas por las instituciones del Estado. Estas instituciones se rigen sobre las recomendaciones de la OMM, tanto en la instalación como en mediciones. De lo mencionado se puede decir que los datos utilizados son de la misma naturaleza y que han sido obtenidos bajo los mismos criterios y procedimientos. Los pasos que se han seguido para llevar adecuadamente los cálculos y resultados comprenden cinco etapas importantes:

\section{Recopilación de datos}

Esta etapa consiste en recopilar las series de datos de lluvia diaria para todo el país. Las instituciones que manejan las estaciones medidoras de la lluvia son: Dirección de Recursos Hídricos (DGRH) perteneciente a la Secretaria de Recursos Naturales, Servicio Meteorológico Nacional (SMN), Empresa Nacional de Energía Eléctrica (ENEE), Servicio Autónomo Nacional de Acueductos y Alcantarillados (SANAA) y Universidad Nacional Autónoma de Honduras (UNAH). 


\section{Selección de estaciones mediante el análisis de los datos}

El análisis para sustentar la calidad de datos fue el método de análisis de doble masa, aplicado a nivel de lluvia diaria. Esto da un mejor panorama de análisis de calidad, puesto que se trabaja a una escala pequeña. Este método será aplicado tanto a nivel puntual como espacial, con el fin de analizar los datos al interior mismo de la variable y también el comportamiento con respecto a las otras estaciones de lluvia.

\section{Selección de estaciones como muestra para la investigación}

De los resultados de las etapas 1 y 2 se seleccionaron las estaciones y el tamaño de serie de datos que servirían de muestra para la investigación.

\section{Análisis estadístico de las muestras}

El análisis estadístico se aplicó para determinar el comportamiento lineal de la tendencia (regresión lineal) de la lluvia diaria, analizada por periodos de días de 3, 5, $10,20,30,90,180,365,1025,1825$ y 3650 . La lluvia promedio fue calculada mediante la media aritmética y la media móvil. También el mismo análisis estadístico mencionado fue aplicado para determinar la tendencia lineal de los días sin lluvia para el periodo de 365 días.

\section{Generación de histogramas y mapas geográficos de tendencia}

A partir de las bondades del ARGIS se generaron mapas geográficos de tendencia.

\section{APLICACIÓN Y RESULTADOS}

\section{Recolección de datos}

Los datos recopilados corresponden a lecturas diarias de lluvia obtenidas de diferentes instituciones del Gobierno hondureño que manejan las estaciones de lluvia del país. Estas instituciones son la ENEE, SANAA, SERNA y SMN. El cuadro 1 presenta un resumen de las estaciones obtenidas por institución y el mapa 1 presenta la ubicación geográfica de las estaciones en las cuales se pudo obtener registros de lluvia diaria y su antigüedad en años. 


\section{Cuadro 1. Cantidades de estaciones medidoras de lluvia diaria}

\begin{tabular}{|l|c|}
\hline Institución & $\begin{array}{l}\text { Cantidad de estaciones } \\
\text { obtenidas por } \\
\text { institución }\end{array}$ \\
\hline ENEE & 80 \\
\hline SERNA & 89 \\
\hline SANAA & 11 \\
\hline UNAH & 1 \\
\hline SMN & 24 \\
\hline Total & $\mathbf{2 0 5}$ \\
\hline
\end{tabular}

Fue posible disponer de datos de lluvia diaria de 205 estaciones, las que en su mayoría son manejadas por la ENEE y la SERNA

\section{Selección de estaciones a partir del análisis de calidad de datos}

En esta etapa se seleccionaron las estaciones finales con las cuales se realizó el análisis de la tendencia de precipitación a nivel diario. El proceso de selección comprendió varias etapas o filtros, en los cuales se verificó no solo la cantidad de datos, sino también su calidad mediante análisis estadísticos de doble masa: agrupación de estaciones, verificación de registro mínimo de años, análisis preliminar de la serie de datos, análisis de doble masa puntual y análisis de doble masa espacial.

\section{Agrupación de estaciones}

Todas las estaciones recolectadas fueron agrupadas tomando en cuenta la topografía, la zona climática y las condiciones de barlovento y sotavento, obteniendo 30 grupos de estaciones. El mapa 2 muestra la distribución geográfica de los mismos.

\section{Verificación de registro de años de datos}

Inicialmente se seleccionaron 30 años de datos de lluvia como longitud mínima; pero debido a que esta condición descartaba 96 estaciones, que equivale al $46 \%$ del total, se tomó la decisión de escoger como longitud mínima 20 años. El cuadro 2 muestra los porcentajes de estaciones que disponen de registros mayores o iguales de 20 años y también los menores de 20 años. Del proceso de verificación de registro mínimo de años resultaron seleccionadas 167 estaciones que fueron sometidas a la etapa de análisis de doble masa. El mapa 3 muestra en color azul la distribución geográfica de las estaciones con registros mayores o iguales a 20 años. 
Mapa 1. Ubicación espacial de las estaciones medidoras de lluvia diaria

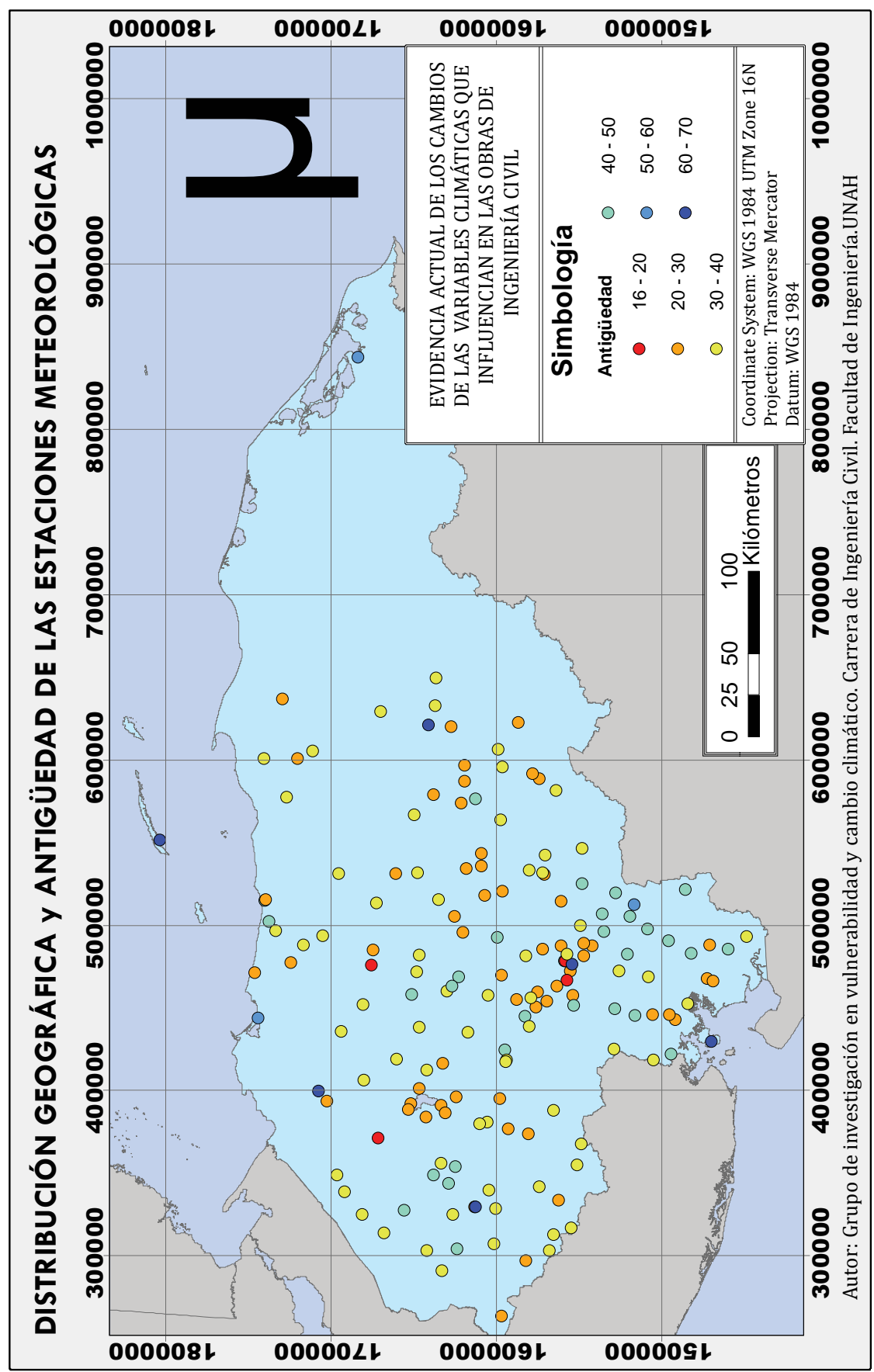

Fuente: Roberto Avalos (2015) 
Mapa 2. Ubicación geográfica de los grupos de estaciones

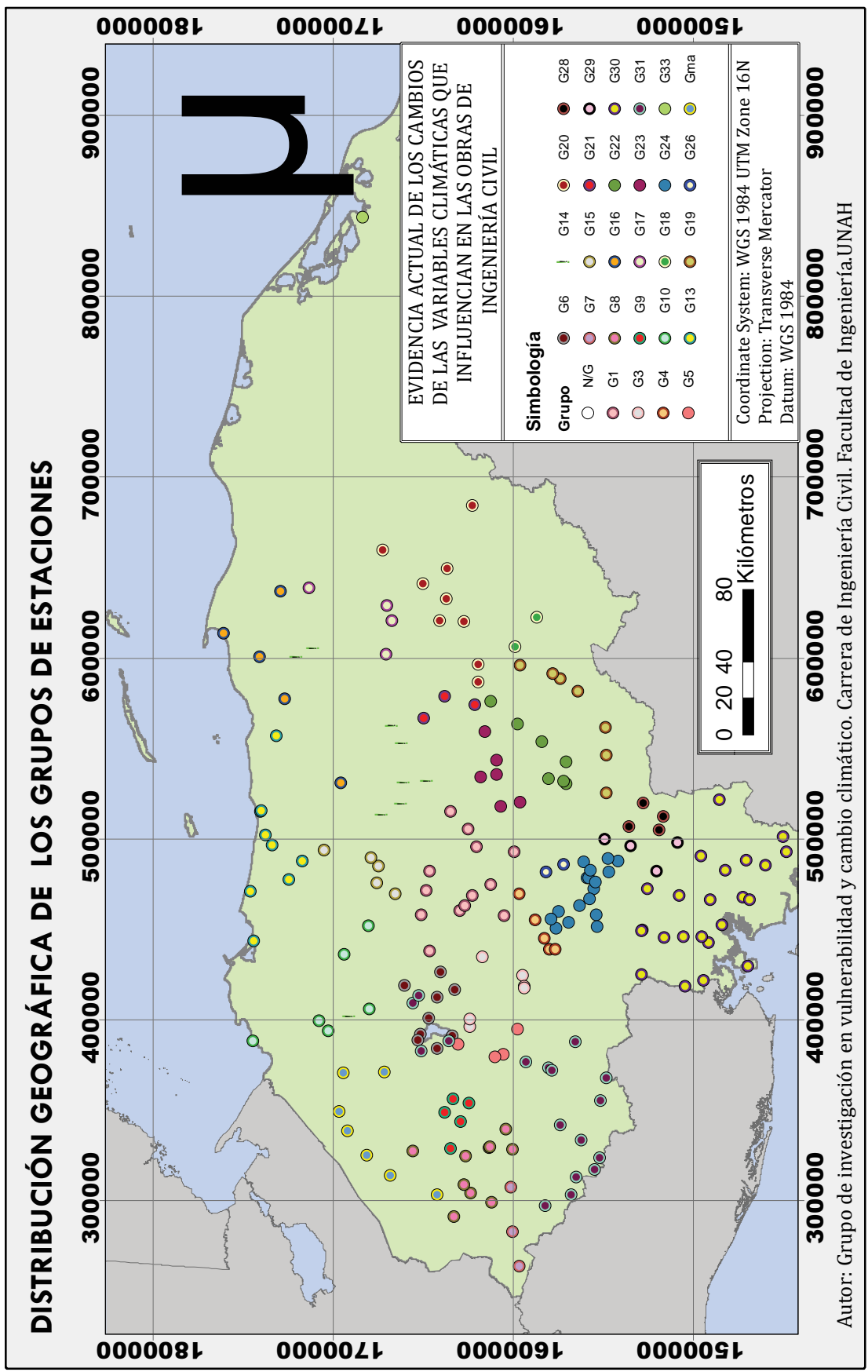

Fuente: Roberto Avalos (2015) 
Mapa 3. Ubicación de las estaciones con registro mayor de 20 años

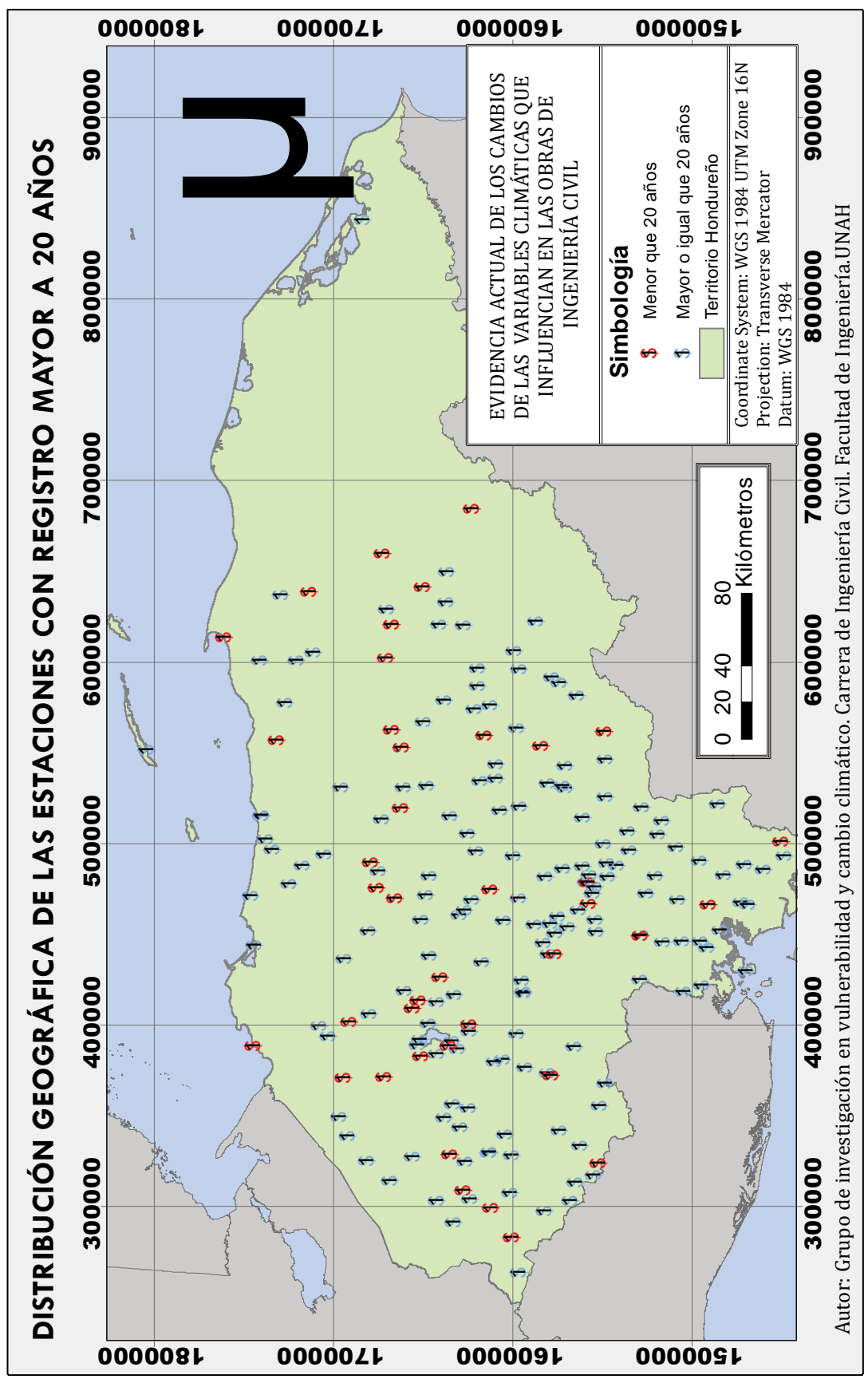

Fuente: Roberto Avalos (2015) 


\section{Cuadro 2. Listado de la cantidad de estaciones seleccionadas}

\begin{tabular}{|l|l|l|}
\cline { 2 - 3 } \multicolumn{1}{c|}{} & $\begin{array}{l}\text { Cantidad de } \\
\text { estaciones }\end{array}$ & Porcentaje \\
\hline $\begin{array}{l}\text { Estaciones con registro menor que 20 } \\
\text { años }\end{array}$ & 38 & 18.54 \\
\hline $\begin{array}{l}\text { Estaciones con registro mayor o igual } \\
\text { que 20 años }\end{array}$ & 167 & 81.46 \\
\hline Total & 205 & 100.00 \\
\hline
\end{tabular}

El cuadro 2 muestra que de las 205 estaciones disponibles, solo 167 tienen datos iguales o mayores a 20 años.

\section{Análisis preliminar de datos}

Antes de someter los datos al análisis de doble masa, se realizó una rápida verificación en lo concerniente a errores de registro, a información incompleta y a datos repetidos. En este caso se descartaron 7 estaciones por tener varios años repetidos.

4. Análisis de doble masa puntual

El análisis de doble masa fue aplicado a las estaciones seleccionadas. Los resultados se observan en el cuadro 3.

\section{Cuadro 3. Resumen de los resultados de doble masa puntual}

\begin{tabular}{|l|l|l|}
\cline { 2 - 3 } \multicolumn{1}{c|}{} & $\begin{array}{l}\text { Cantidad de } \\
\text { estaciones }\end{array}$ & Porcentaje \\
\hline Aprobaron el análisis de doble masa individual & 136 & 84.47 \\
\hline $\begin{array}{l}\text { No aprobaron el análisis de doble masa } \\
\text { individual }\end{array}$ & 25 & 15.53 \\
\hline TOTAL & 161 & 100.00 \\
\hline
\end{tabular}

El cuadro 3 muestra que el $84.47 \%$ aprobaron el análisis de doble masa puntual. 
Mapa 4. Distribución geográfica de las estaciones finalmente seleccionadas

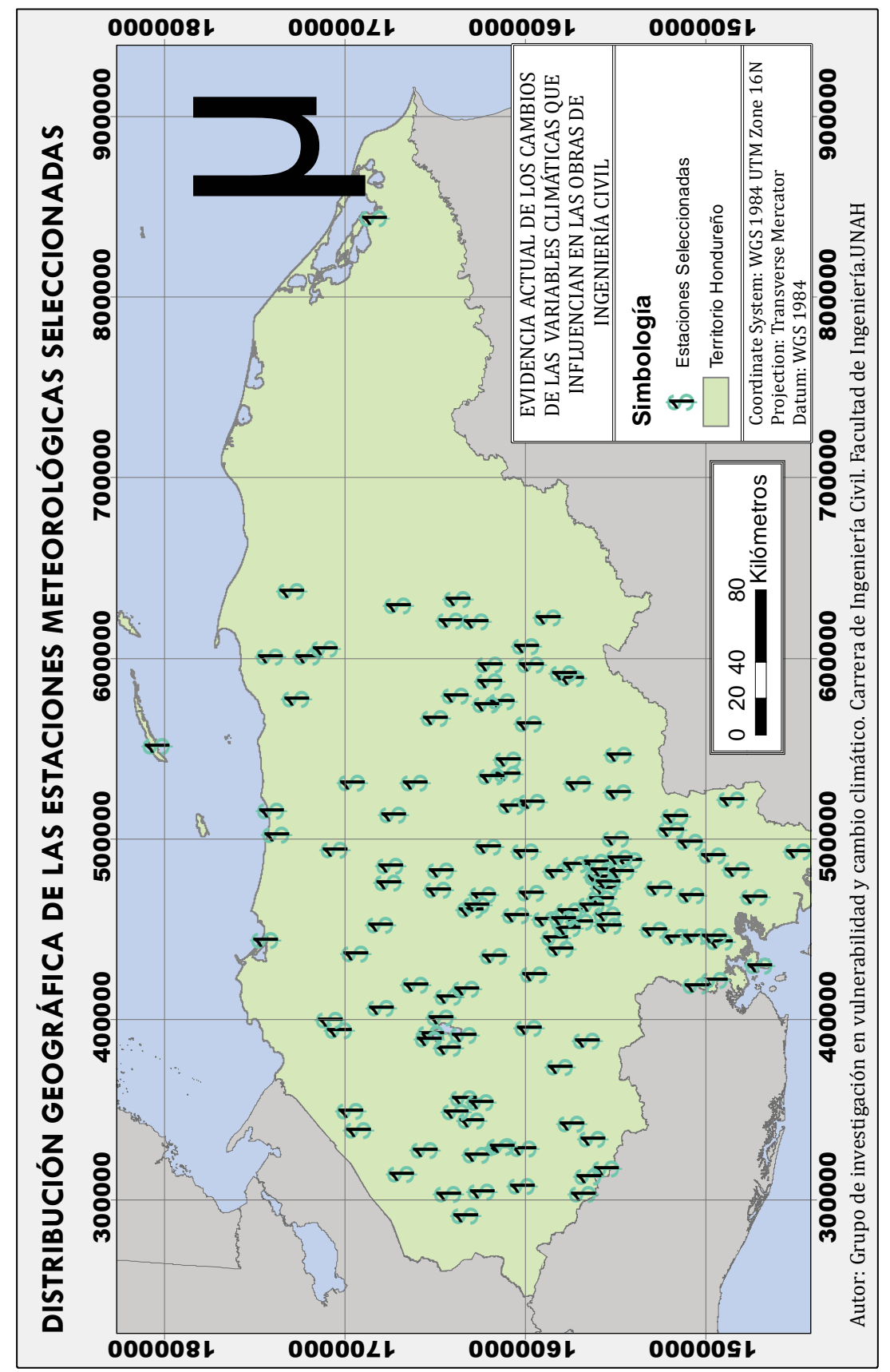

Fuente: Roberto Avalos (2015) 


\section{Análisis de doble masa espacial}

La aplicación del método de doble masa a nivel espacial fue realizada con base a los grupos de estaciones antes mencionados. El cuadro 4 muestra los resultados.

Cuadro 4. Resumen de la aplicación de doble masa espacial

\begin{tabular}{|l|l|l|}
\cline { 2 - 3 } \multicolumn{1}{c|}{} & $\begin{array}{l}\text { Cantidad de } \\
\text { estaciones }\end{array}$ & Porcentaje \\
\hline $\begin{array}{l}\text { Aprobaron el análisis de doble masa } \\
\text { espacial }\end{array}$ & 129 & 94.85 \\
\hline $\begin{array}{l}\text { No aprobaron el análisis de doble masa } \\
\text { espacial }\end{array}$ & 7 & 5.15 \\
\hline Total & 136 & 100 \\
\hline
\end{tabular}

El $94.85 \%$ aprobaron el análisis de doble masa espacial. El mapa 4 muestra la distribución geográfica de las estaciones finalmente seleccionadas

Análisis estadístico de regresión aplicado a las series de lluvia diaria de las estaciones seleccionadas (Iluvia promedio=f (tiempo))

Con las series de lluvia diaria de las estaciones seleccionadas de la etapa anterior se determinó la lluvia promedio para los periodos en días de 3, 5, 10, 20, 30, 90, 180, $365,1095,1825,3650$. Promedios obtenidos por la media aritmética y la media móvil, valores que fueron graficados y ajustados a la regresión lineal. Dado que se obtuvieron una gran cantidad de figuras de tendencia, se consideró importante mostrar por lo menos un ejemplo de esto (ver figuras 1 y 2 ).

Figura 1. Estación Coray, caso media aritmética

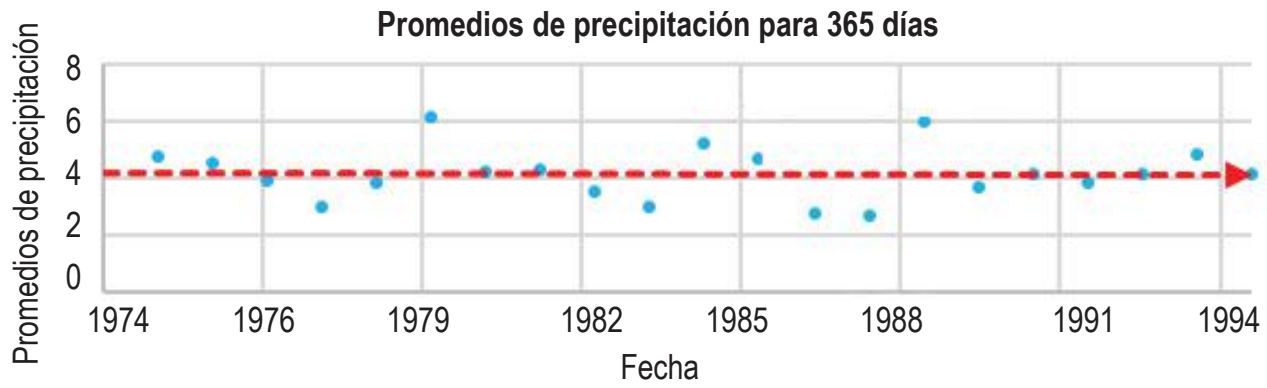

$$
Y=-1 E-05 x+4.5772 \quad R^{2}=0.001
$$




\section{Figura 2. Estación Coray, caso media móvil}

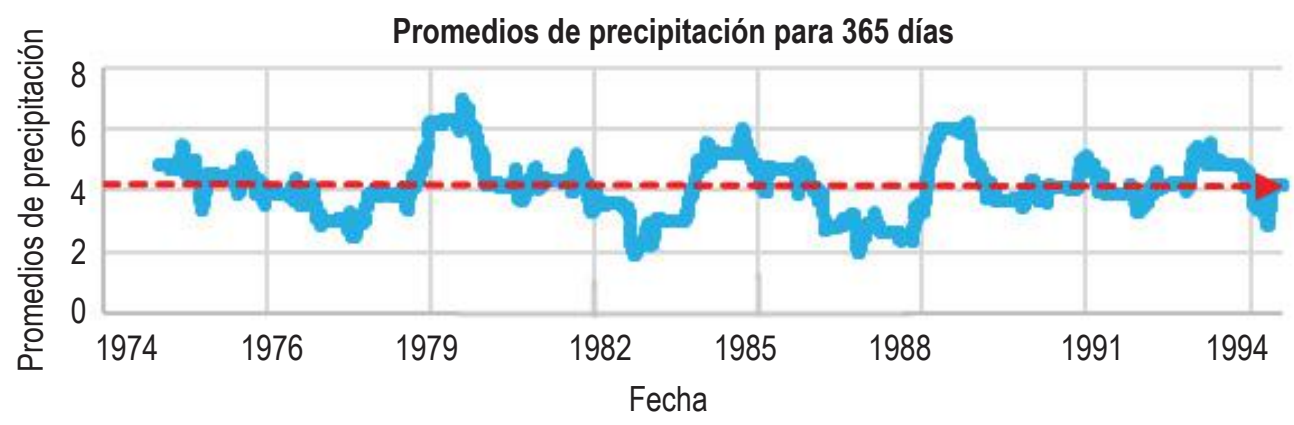

$Y=-9 E+4.4435 \quad R^{2}=0.0004$

Con el fin de tener otra variable que apoye y sustente los resultados de la tendencia de las regresiones se realizó el análisis de tendencia de los días sin lluvia para el periodo de 365 días de las estaciones ya seleccionadas. Lo cual significa que si los días sin lluvia aumentan, es probable que la cantidad de lluvia tienda a la disminución. La figura 3 muestra el ejemplo para la estación Coray.

Todos los resultados obtenidos de tendencia (magnitud y simbología de la pendiente) fueron representados en mapas geográficos mediante el uso del ARGIS y de la interpolación de Kriging. Los mapas diseñados son presentados en los mapas del 5 al 9 . Solamente se presentan los mapas a partir del periodo de tiempo de 10 días, a fin de evitar el diseño de muchos mapas.

Figura 3. Estacion Coray. Tendencia de días sin Iluvia

Cantidad de días sin lluvias, Coray (1974-1994)

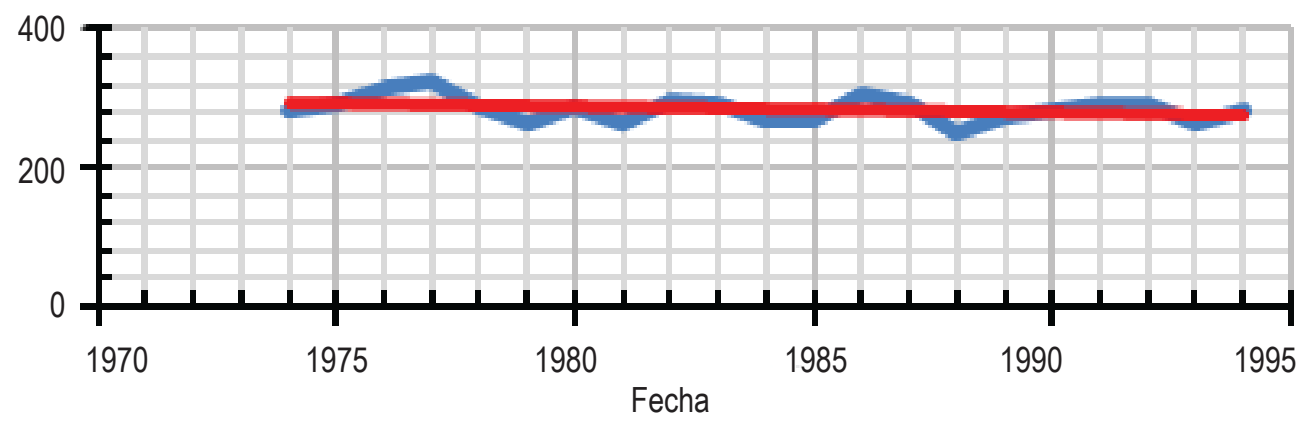

$Y=-0.9 x+20701.2 \quad R 2=0.0971$ 


\section{Mapa 5. Tendencia de la lluvia método aritmético para estaciones con datos entre 20 y 30 años}

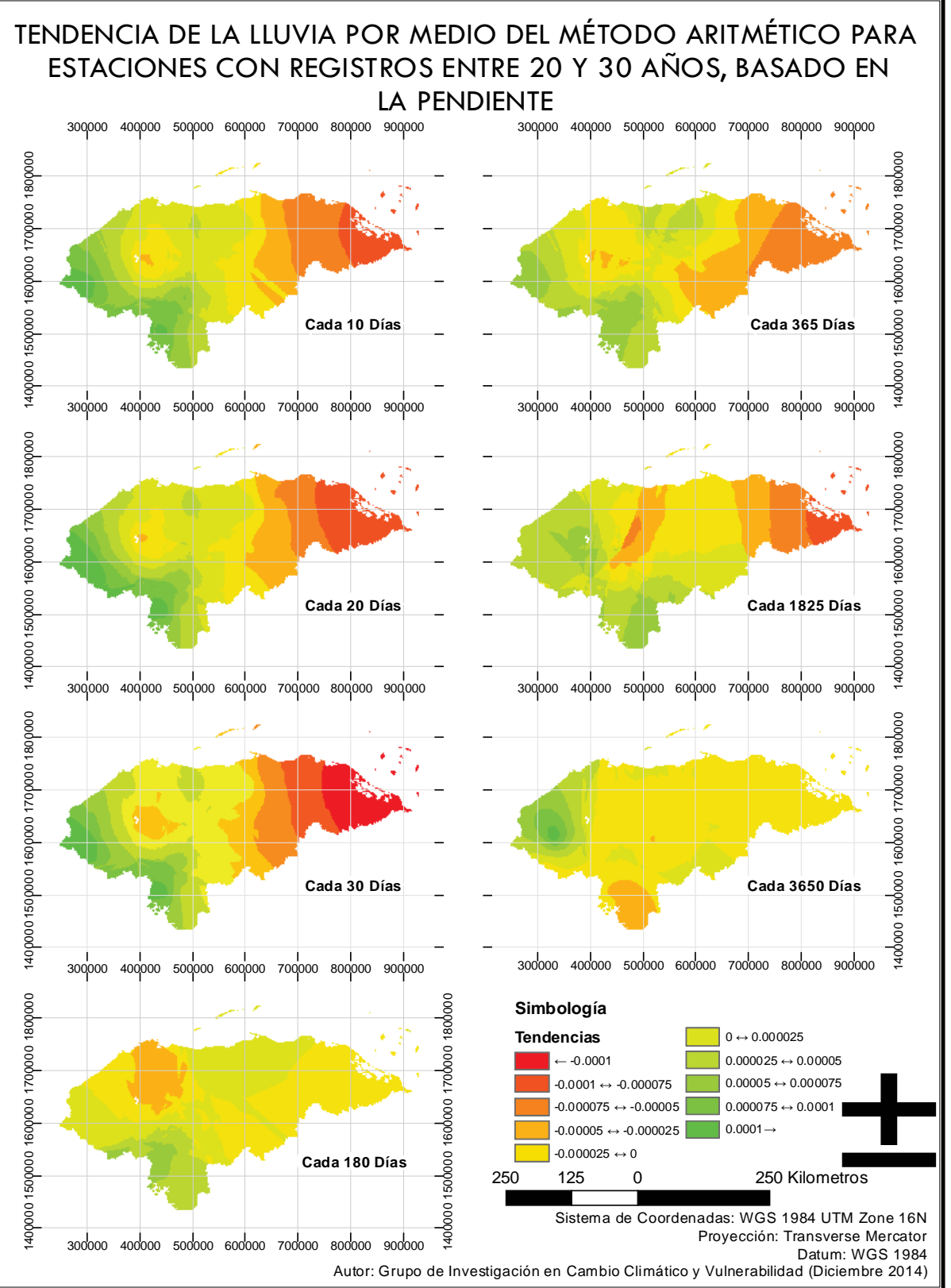

Fuente: Roberto Avalos (2015) 


\section{Mapa 6. Tendencia de la lluvia método aritmético para estaciones con datos mayores de 30 años}

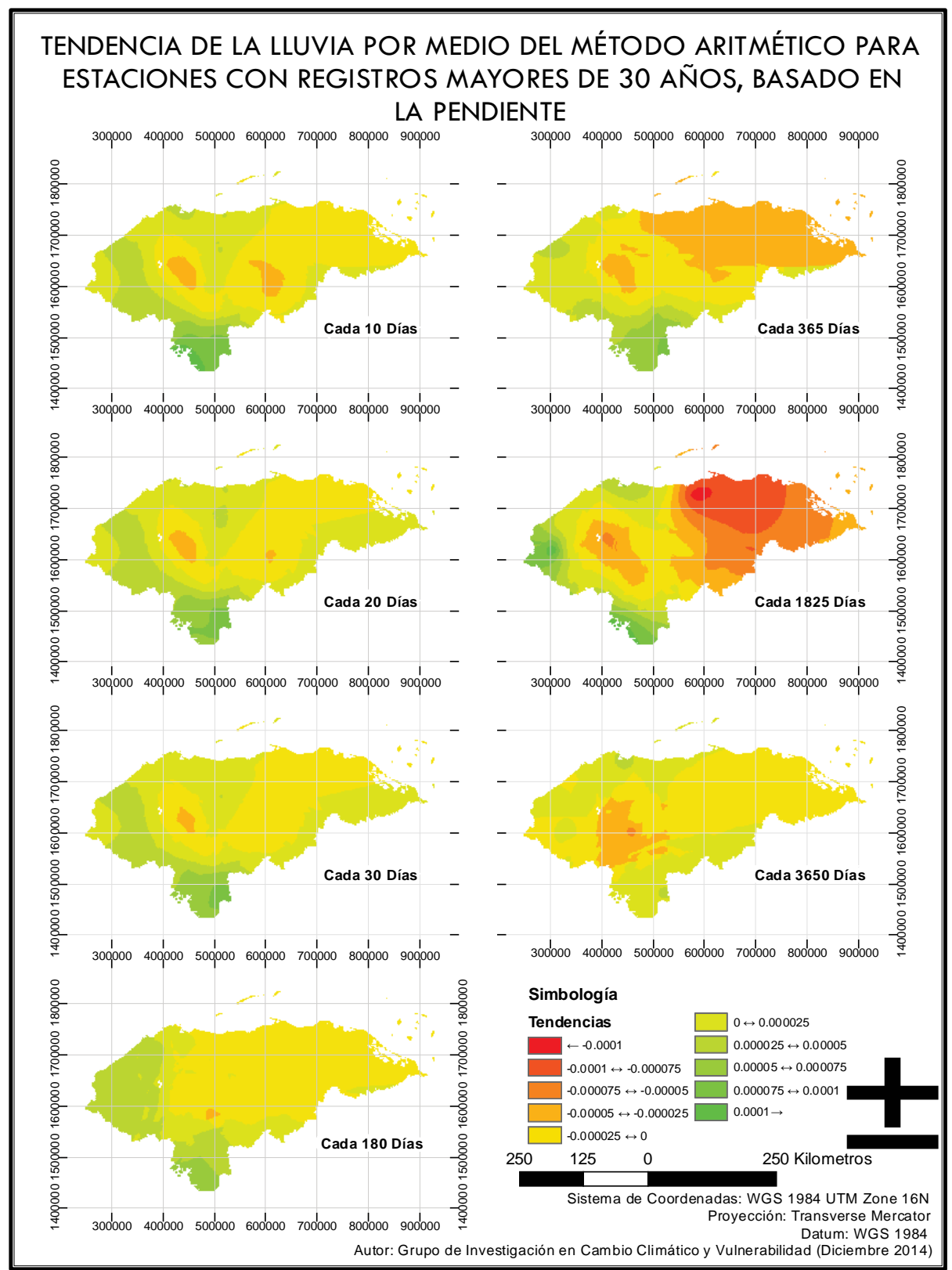

Fuente: Roberto Avalos (2015) 


\section{Mapa 7. Tendencia de la lluvia método media móvil para estaciones con datos entre 20 y 30 años}

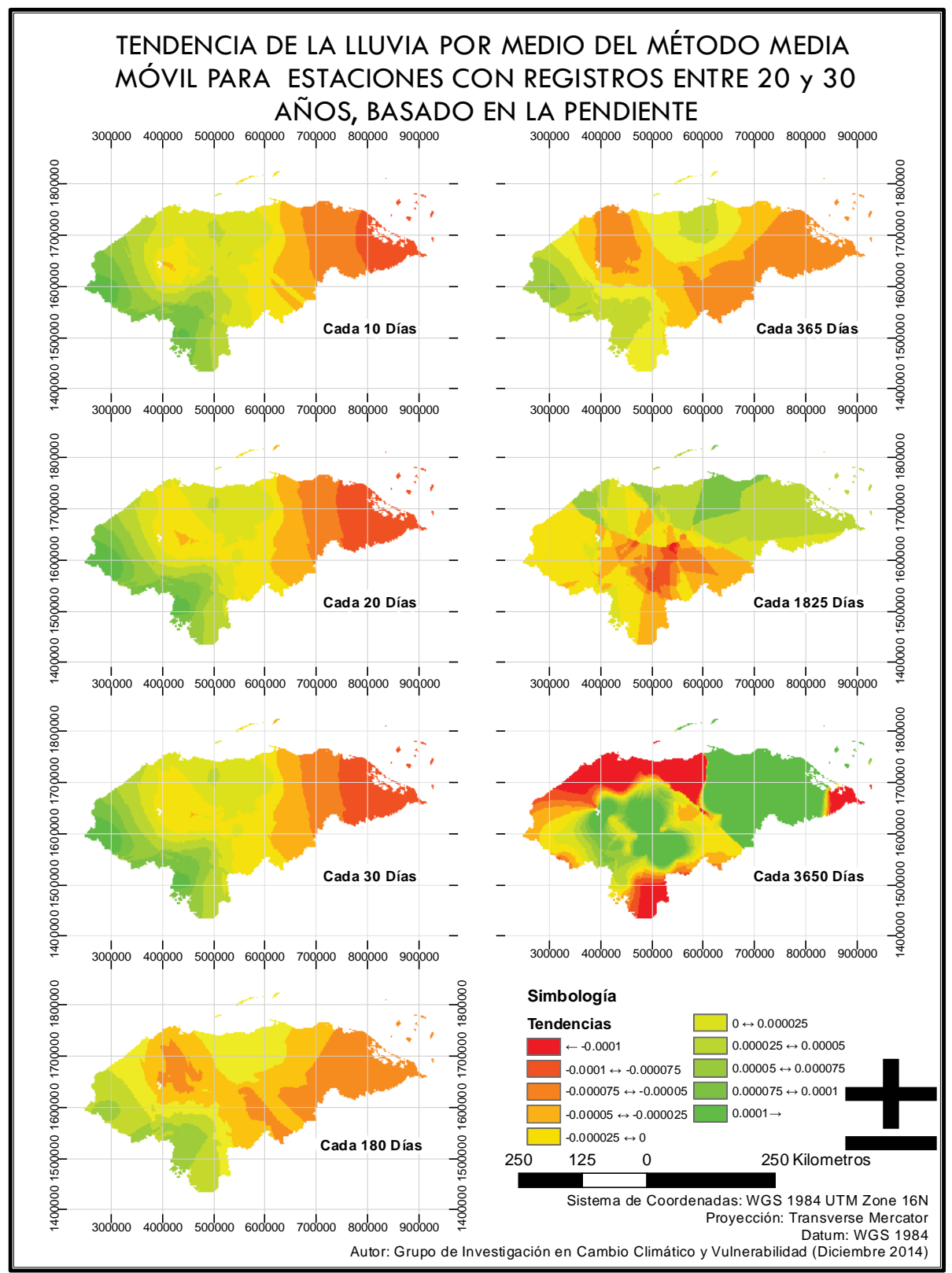

Fuente: Roberto Avalos (2015) 


\section{Mapa 8. Tendencia de la lluvia método media móvil para estaciones con datos mayores de 30 años}

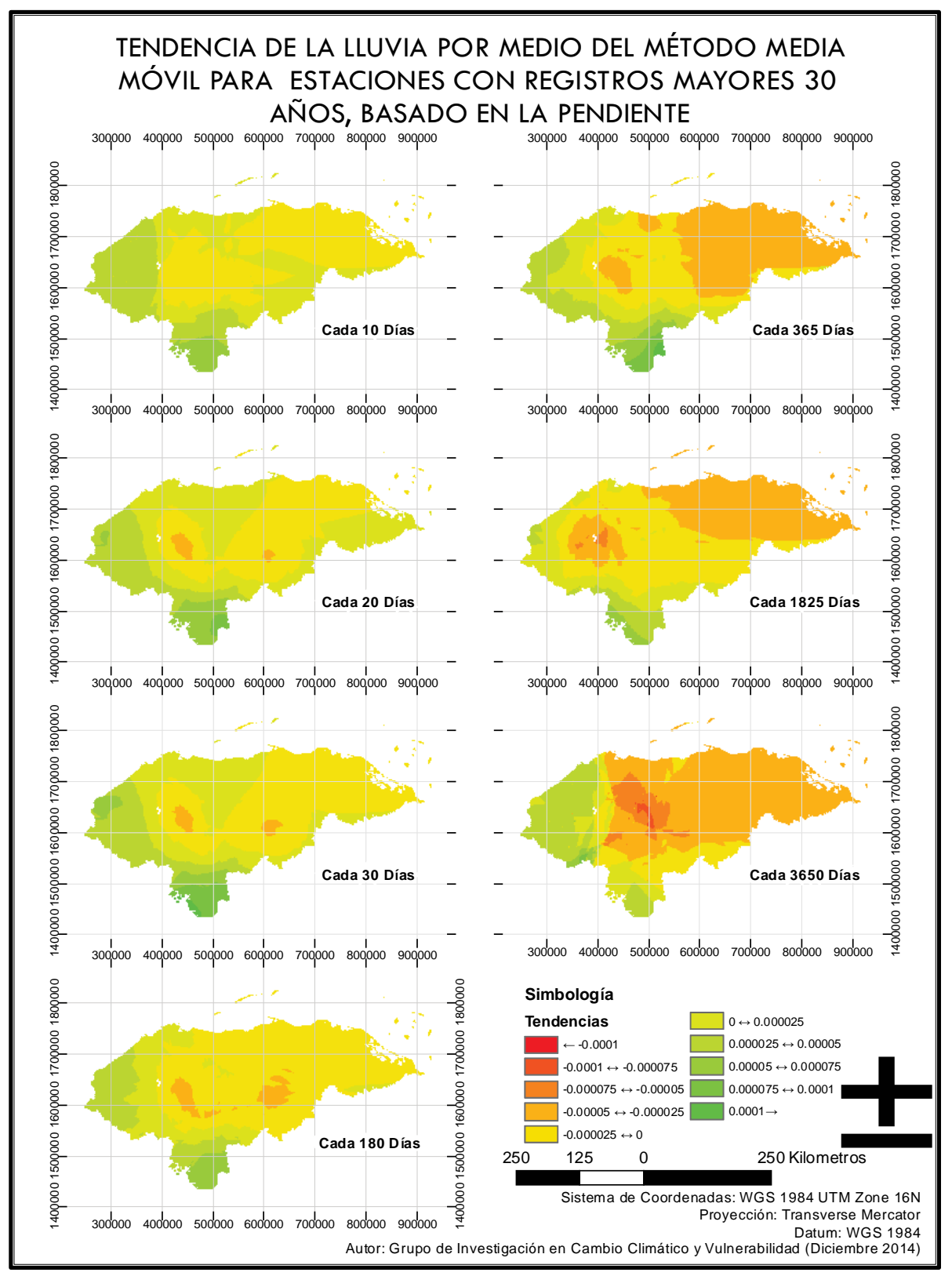

Fuente: Roberto Avalos (2015) 
Mapa 9. Tendencia de los días sin Iluvia

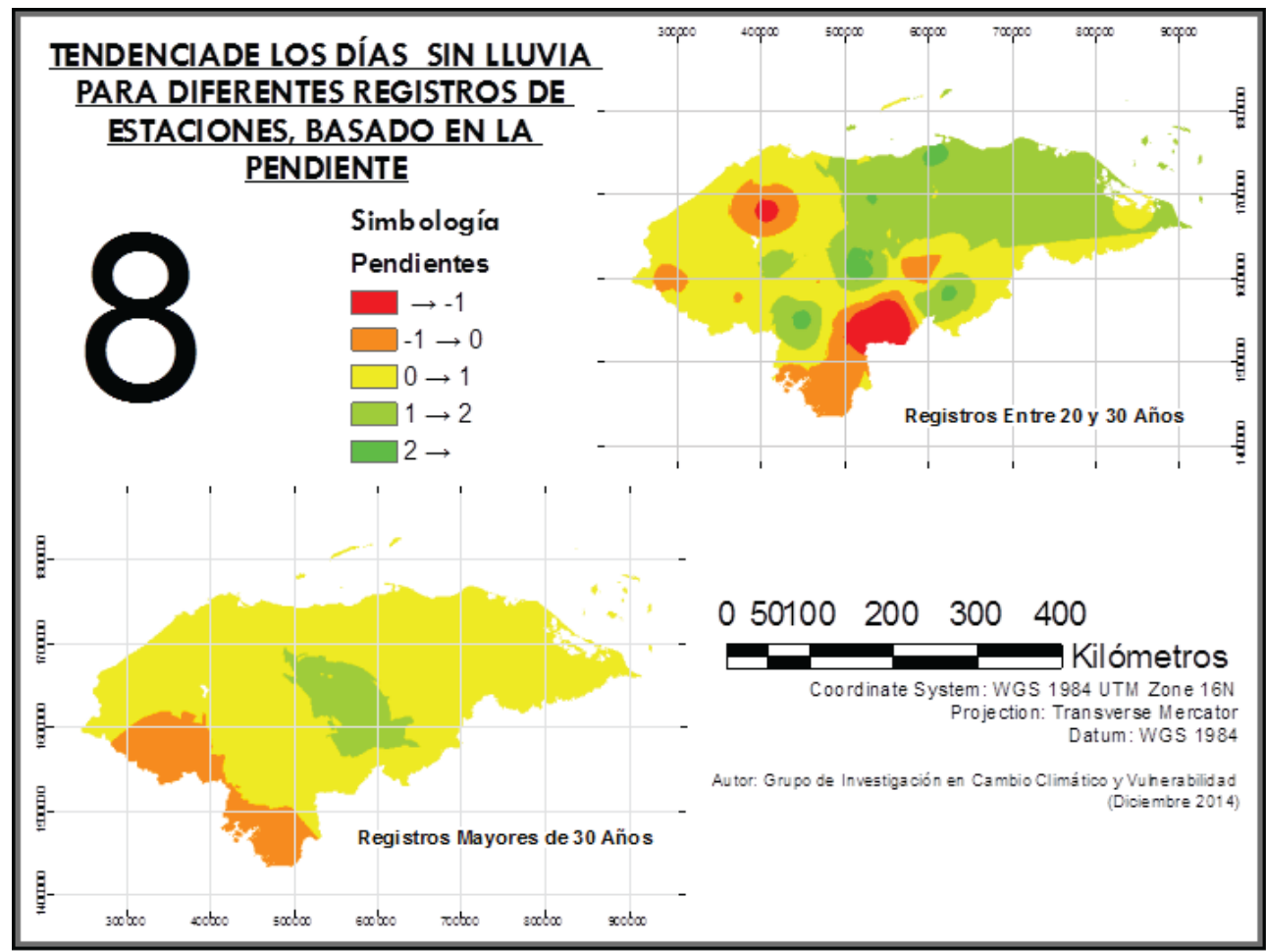

Fuente: Roberto Avalos (2015)

\section{DISCUSIÓN DE RESULTADOS}

La etapa de recolección de datos de lluvia diaria de las estaciones que manejan las instituciones gubernamentales en todo el territorio hondureño, permitió disponer de 205 estaciones. Con estos datos se realizó la primera depuración, eliminando las series que tienen menos de 20 años de lluvia observada, quedando de esto 167 series. El proceso de análisis de calidad de datos a que fueron sometidas todas las series de lluvia mermaron el número de estaciones disponibles, quedando finalmente 130 estaciones, que representa 1 estación por cada $865 \mathrm{~km} 2$. Cabe mencionar que en el presente análisis y discusión de resultados no se tomó en cuenta la región de Gracias a Dios (UTM longitud E mayor de $700000 \mathrm{~m}$ ) a pesar de que los resultados de la interpolación son proyectados hasta dicha región; debido que solo se dispuso de la estación de Puerto Lempira (ver mapa 1). 
Los mayores problemas en el proceso de análisis de calidad no fueron tanto por la incongruencia de datos, sino debido a los vacíos al interior de las series por falta de medición.

Los cálculos de tendencia de la lluvia fueron realizados para los periodos de tiempo, en días de $3,5,10,20,30,90,180,365,1025,1825$ y 3650. Por cuestión de facilidad de presentación, los resultados se muestran para los periodos de 10 días en adelante.

En general, los resultados muestran que las evidencias de aumento o disminución de la lluvia diaria a partir de las series seleccionadas y analizadas en pasos de tiempo diferentes no están bien definidas, ya que las pendientes y coeficientes de correlación lineal tienen valores que son muy bajos. Las pendientes oscilan entre -0.000025 y 0.00025 . Hay regiones en donde aparentemente se observa alguna evidencia de un aumento, otras donde parece que hay disminución y otras cuya pendiente es tan pequeña que reina la incertidumbre. La tendencia lineal de aumento o disminución de la lluvia parece ser no significativa durante los periodos de análisis investigado. Esto concuerda con lo mencionado por Brunetti y otros (2006), quienes investigaron con datos mensuales de los dos últimos 200 años para toda Italia y en donde manifiestan una tendencia decreciente de la variable lluvia ( $5 \%$ ), aunque baja y raramente significativa.

Los resultados de la aplicación de la metodología han sido representados espacialmente sobre el territorio hondureño. Los resultados de la tendencia, aunque aparentes, muestran tendencia al aumento o disminución. Tales resultados se interpretan a continuación.

En el caso de series con longitudes mayores de 30 años, los resultados tanto de promedio aritmético como el de la media móvil muestran una tendencia al aumento de la lluvia para el $65 \%$ del territorio. Pero, este $65 \%$ va disminuyendo conforme aumenta el periodo de tiempo, al grado que llega a invertirse dicho porcentaje; es decir, 65 $\%$ del área de país tiene tendencia a la disminución de la lluvia. A pesar de estos cambios se observan regiones que mantienen la tendencia de aumento, como en los departamento de Choluteca, Valle, La Paz, El Paraíso sur, Lempira, Intibucá, Ocotepeque, Copán, Cortés, Atlántida, gran parte de Santa Bárbara y sur de Francisco Morazán. Dentro de las regiones que mantienen la tendencia de disminución de la lluvia están los departamentos de Comayagua (centro y norte), norte y centro de Francisco Morazán, parte este del lago de Yojoa, Olancho, este de Yoro y Colón.

Para el caso de las longitudes de series entre 20 y 30 años, los resultados muestran 
que hasta el periodo de tiempo de 30 días, estos son muy parecidos al caso de series mayores de 30 años. Honduras, al igual que los otros países de Centroamérica, no dispone de series largas como los países de Europa, EE.UU., Canadá, Rusia y otros. Incluir series cortas con series largas de más de 30 años dificulta la interpretación.

Los resultados obtenidos de la presente investigación realizada sobre la tendencia de la lluvia utilizando las series disponibles del territorio de Honduras, caracterizado como país tropical, muestran que la influencia del cambio climático en la variable lluvia como cantidad medida en el terreno y utilizando lluvia de resolución diaria no está todavía bien marcada; solamente hay indicios de aumento o disminución.

En lo concerniente a los resultados obtenidos y presentados de manera espacial, tanto para el caso de la media aritmética como para la media móvil, ambos dan resultados que no son $100 \%$ iguales; en algunos resultados aparentemente son similares y en otros no. En cuanto a los resultados obtenidos de la tendencia de los días sin lluvia para un paso de tiempo de 365 días y para las series mayores de 30 años, muestran que estas son negativas para las regiones que cubren los departamentos de Choluteca, Valle, La Paz, sur de Intibucá y gran parte de Lempira. Por otra parte, las regiones comprendidas entre la parte oeste de Olancho, Comayagua este y Yoro oeste tienen una tendencia al aumento de los días sin lluvia.

Observar una tendencia de aumento o disminución de los días sin lluvia no significa estrictamente una tendencia también de aumento o disminución de la cantidad de lluvia. Si cruzamos la tendencia de las cantidades de lluvia con la tendencia de días sin lluvia ambas para 365 días, nos encontramos con la sorpresa de que la región de Departamentos como Choluteca, Valle, La Paz, Sur de Intibucá y gran parte de Lempira coinciden con la tendencia aparentemente positiva; en cambio la parte Oeste de Olancho, Este de Comayagua y Suroeste de Yoro coinciden con la tendencia aparentemente negativa.

\section{CONCLUSIONES}

Con series de lluvia diaria medida en el terreno y con longitudes mayores de 30 años de datos (de 2013 a 1944), manejadas por instituciones gubernamentales de Honduras, país centroamericano y de clima se concluye lo siguiente: 
1. En la influencia del cambio climático en la lluvia a resolución diaria y como cantidad, todavía no se ha podido encontrar evidencias bien definidas sobre la tendencia lineal de aumento o disminución en la magnitud de dicha variable. Las pendientes de las tendencias son muy bajas para definir significativamente el aumento o disminución de la lluvia.

2. Dentro de lo aparente se puede decir que la región comprendida por los departamentos de Choluteca, Valle, La Paz, el sur de El Paraíso, Lempira, Intibucá, Ocotepeque, Copán, parte centro y norte de Cortés, oeste y centro de Atlántida, gran parte Santa Bárbara y sur de Francisco Morazán muestran una tendencia muy pequeña de aumento de la lluvia. La región comprendida por los departamentos de Comayagua (centro y norte), norte y centro de Francisco Morazán, parte este del lago de Yojoa, sur de Cortés, centro y norte de El Paraíso, Olancho, este de Yoro y Colón, muestran una tendencia a la disminución de la lluvia.

3. Para el caso del periodo de 365 días, la tendencia de disminución de días sin lluvia apoya la tendencia positiva de la lluvia para los departamentos de Choluteca, Valle, La Paz, sur de Intibucá y gran parte de Lempira. De igual forma, la tendencia positiva de los días sin lluvia para la parte oeste de Olancho, este de Comayagua y suroeste de Yoro.

\section{RECOMENDACIÓN}

Continuar la investigación para seguir buscando evidencia de la influencia del cambio climático en la variable lluvia en su dimensión de intensidad, duración y frecuencia. Asimismo, continuar con las otras variables climáticas como temperatura (media, máxima y mínima), humedad relativa, viento, evaporación, etc.

\section{BIBLIOGRAFÍA}

Argeñal, F. (2010). Variabilidad climatica y cambio climático en Honduras. Tegucigalpa: Ed. SERNA PNUD.

Arthenus, S. (1896). Efecto de la duplicacion del óxido de carbono en la atmósfera. Suecia.

Brunetti, M.; Mauger, M.; Monti, F. \& Nannia, T. (2006). Temperature and Precipitation variability in Italy in the last two centuries from homogenised instrumental time series. International Journal of Climatology. Volumen 26, numero 3, pag: 345-381. 
Easterling, D. (2000). Observed variability and trends in extreme climate events:a brief review. Bulletin American Meterological Society, Numero 81, 417-425.

Environnement Canadá. (2010). Bulletin des tendences et de variations climatique-ete 2010. Québec: Environnement Canadá, Gouvernemet du Canadá.

Fourier, J. (1827). Memoire sur la temperature du globe terrestre et espaces. France. J. SCciene, 32, 1-20.

Grimm, A. (1999). Climate Variability in Southern South America Associated with El Niño and La Niña Events. Argentina: J. Clim, 13,35-58.

Hogbom, A. (1890). Ciclo del carbono, estimación de la producción de carbono de fuente industrial. Suecia.

Houghton. (2001). Climate change. The scientific basic. USA, New York. Published for the Intergovernmenta Panel on Climate Change. Cambridge University Press.

Jones, P. (1999). Climates changes scenarios for the United Kingdom. U.K. Climate Impacts Programme. United Kingdom.

Leverman, D. \& O'Brien, K. (1991). Global Warming and Climate Change in Mexico. Global Environmental Change, Volumen 1, Numero 5, 351-364.

Manabe, S. \& Wetherald, R. (1967). Thermal Equilibrium of the Atmosphere with a Given Distribution of Relative Humidity. Journal of the Atmospheric Sciences, Volumen 24, Numero 3, 241-259.

OMM. (1988). Riesgo del cambio climático provocado por la actividad humana. Ginebra.

Royal-Meteorological-Society. (2002). Daily dataset of 20Th-Century surface air temperature and precipitation series for the European Climate Assessment. Wiley Intersciences. Royal Netherlands Meteorological Institute. Bulletin of American Meteorological Society, 78.

Trenberth, K. (2007). Observation: Surface and Atmospheric Climate Change. Climate Research. Ambenje -IPCC, nldr.library.ucar.edu.

Tyndall, J. (1864). Contributions to molecular physics. London. rstl.royalsocietypublishing.org.

Zúniga, Edgardo, 1989 y 1990. Las modalidades del Clima en Honduras y las Variantes del Clima de Honduras. Obras publicadas por Editorial Guaymuras y Banco Central de Honduras. 